\title{
Deep neural architectures for mapping scalp to intracranial EEG
}

\author{
Andreas Antoniades \\ Department of Computer Science, University of Surrey, Guildford, Surrey, GU2 7XH, United Kingdom \\ E-mail: a.antoniades@surrey.ac.uk \\ Loukianos Spyrou \\ School of Engineering, University of Edinburgh, EH9 3FB, United Kingdom \\ E-mail: l.spyrou@ed.ac.uk \\ David Martin-Lopez \\ Kingston Hospital NHS FT, London, SE5 9RS, UK and King's College London, WC2R 2LS, UK \\ E-mail: david.martinlopez@kcl.ac.uk) \\ Antonio Valentin \\ King's College Hospital, London, UK and King's College London, WC2R 2LS, UK \\ E-mail: antonio.valentin@kcl.ac.uk \\ Gonzalo Alarcon \\ Hamad Medical Corporation, Doha, Qatar and King's College London, WC2R 2LS, UK \\ E-mail: galarcon@aol.com \\ Saeid Sanei \\ Department of Computer Science, University of Surrey, Guildford, Surrey, GU2 7XH, United Kingdom \\ E-mail:s.sanei@surrey.ac.uk \\ Clive Cheong Took \\ Department of Computer Science, University of Surrey, Guildford, Surrey, GU2 7XH, United Kingdom \\ E-mail: c.cheongtook@surrey.ac.uk
}

\begin{abstract}
Data is often plagued by noise which encumbers machine learning of clinically useful biomarkers and EEG data is no exemption. Intracranial EEG data enhances the training of deep learning models of the human brain, yet is often prohibitive due to the invasive recording process. A more convenient alternative is to record brain activity using scalp electrodes. However, the inherent noise associated with scalp EEG data often impedes the learning process of neural models, achieving substandard performance. Here, an ensemble deep learning architecture for non-linearly mapping scalp to intracranial EEG data is proposed. The proposed architecture exploits the information from a limited number of joint scalpintracranial recording to establish a novel methodology for detecting the epileptic discharges from the scalp EEG of a general population of subjects. Statistical tests and qualitative analysis have revealed that the generated pseudo-intracranial data are highly correlated with the true intracranial data. This facilitated the detection of IEDs from the scalp recordings where such waveforms are not often visible. As a real world clinical application, these pseudo-intracranial EEG are then used by a convolutional neural network for the automated classification of intracranial epileptic discharges (IEDs) and non-IED of trials in the context of epilepsy analysis. Although the aim of this work was to circumvent the unavailability of intracranial EEG and the limitations of scalp EEG, we have achieved a classification accuracy of $64 \%$; an increase of $6 \%$ over the previously proposed linear regression mapping.
\end{abstract}


Keywords: interictal epileptic discharge; scalp to intracranial EEG mapping; asymmetric deep learning.

\section{Introduction}

EEG is a brain recording modality that captures the electric activity of the brain ${ }^{1}$. Those electric fields are traditionally measured by scalp electroencephalogram (sEEG) offering a cheap and non-invasive method at the cost of low signal to noise ratio (SNR). sEEG has found a number of applications including brain-computer interface ${ }^{2}$ (BCI) and epilepsy detection $^{3,4}$. For clinical and rehabilitation applications, intracranial EEG (iEEG) is a better alternative in many fields ${ }^{5,6}$, since the effects of background activities, attenuation, and blurring/delay are less prominent than in the SEEG $^{1}$.

Data is often tainted with different kinds of noise, depending on the equipment used to capture it and other external factors. In the context of sEEG data, subjects differ in terms of noise due to variations in neural generation mechanisms originating in the brain cortex that depend on age, gender and medical condition. These factors are often referred to as background EEG and make independent subject monitoring a difficult task. In fact, it has been shown that some intracranial epileptic discharges (IEDs) generated by deep structures may not be visible on the scalp because of the relatively high attenuation of electrical fields due to distance from the source and high attenuation by the skull, essentially masking them beneath the noise floor ${ }^{7,8}$. An example of attenuation of IED, captured by Foramen Ovale (FO) electrodes, evident in the sEEG is shown in Fig. 1. Such attenuation of IED makes it hard for the clinician and the data analyst to label periods of EEG data as IED and non-IED. IED detection techniques have been developed for scalp ${ }^{9,10}$ or intracranial ${ }^{11,12}$ EEG separately, but no joint modalities have been exploited. To address this shortcoming in the literature, we aim to recover iEEG from sEEG by approximating the mapping between these two modalities. To this end, we introduce a non-linear approach to map the scalp signals to their corresponding intracranial activations. Such mapping has been considered before, however, these preliminary findings may not have captured the complexity of the EEG data. For instance, Kaur et $\mathrm{al}^{14}$ investigated the linear mapping of non-epileptic sEEG responses to the concur- rent iEEG via a regression model and subsequently classified through an iEEG classifier. Another work ${ }^{15}$ explored dictionary learning that mapped scalp to intracranial EEG in a within-subject paradigm. These works provided valuable first insights into the problem of mapping scalp to intracranial EEG, yet the techniques were modeled based on a single layer of machine learning, even though the data may be too complex to be learned by such a simple model. On the other hand, deep learning of neural networks has shown that the complexity of the data can be broken down into several lower complexity data through many layers; each of those layers are trained one at a time $^{16}$. For instance, in image processing, the neural network learns rudimentary features such as edges in the first layers, while more complex shapes are learned in deeper layers ${ }^{17}$. Therefore, deep learning is considered for the mapping of sEEG to iEEG to introduce non-linearity and break down the learning process into multiple stages, each performed by a layer.

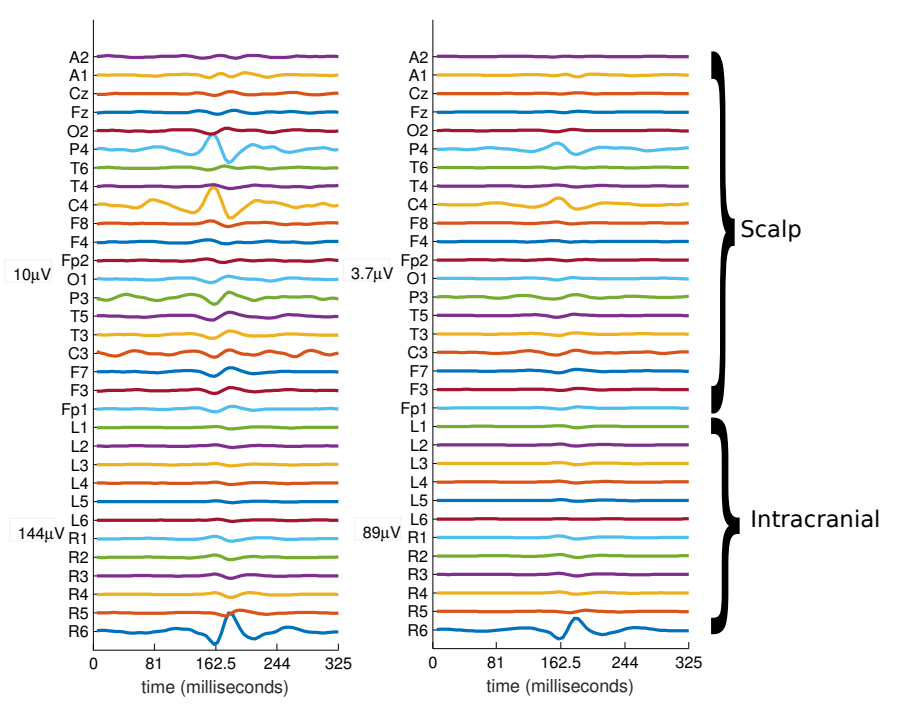

Figure 1. Average amplitudes for scalp and intracranial IEDs $^{13}$ from a subject for segments indicated as visiblescalp (left) and non-visible scalp (right) IEDs. Note the scale difference of the two modalities. The labeling of the intracranial electrodes is indicated as $\mathrm{R} 1$ to $\mathrm{R} 6$ and $\mathrm{L} 1$ to L6 from the deepest to the most superficial right and left side FO respectively. 
Deep learning is a hot topic in image processing, yet it is only starting to emerge in EEG processing. Applications of deep learning in biomedicine include the diagnosis of Alzheimers ${ }^{18}$ and Creutzfeldt-Jakob diseases $^{19}$. Convolutional neural networks (CNNs) have been applied for EEG-based decoding of movement related information ${ }^{20}$, P300 detection for $\mathrm{BCI}^{21}$ and seizure detection ${ }^{22}$. Another example of deep learning in EEG processing was to shed light on the black box nature of neural networks; to make sense of machine learning, it was shown that the learning coefficients of a CNN can converge to the morphology of events of interest in EEG such as the interictal epileptiform discharges ${ }^{23}$.

Deep learning models rely heavily on large volume of data to train. However, EEG datasets rarely contain an adequate number of subjects, which makes it impossible for deep learning to generalize the models in subject independent studies. To address this common shortcoming in EEG datasets, we consider mapping several pseudo-versions of the intracranial EEG data with the aid of noise. A number of articles suggest that incorporating noise in machine learning algorithms can enhance their robustness, especially in the area of image recognition ${ }^{24-26}$. It is in this spirit that we propose a novel mapping algorithm for estimating not one but several pseudoversions of the iEEG for training purposes.

Our approach exploits the unsupervised training capability of autoencoders in a subject independent fashion. Each model learned by each autoencoder, estimates the scalp to intracranial EEG mappings of a specific subject and then creates a new version of the entire dataset based on this mapping. This enables diversity in the data which consolidates the statistical learning of neural networks. To take advantage of the temporal characteristics of the data, a CNN learner is trained to classify each trial of the newly generated datasets as IED and non-IED. After the training is complete, the model closest to that of the patient of interest (unseen test subject) is used to estimate its pseudo-intracranial EEG, which is then classified as IED or non-IED.

As a real world application, this study suggests an unsupervised mapping of sEEG to iEEG in order to improve the SNR and aid clinicians in the identification of IEDs contained in EEG trials. Such a tool has the potential of limiting the amount of raw
EEG recordings, which an expert has to examine in order to distinguish and label such waveforms. Additionally, the use of deep CNNs enables automatic generation of features that can benefit the training of classifiers.

This paper is structured as follows: the problem of mapping scalp to intracranial EEG data is formulated in Section 2, the deep learning models used are introduced in Sections 3, 4 and 5, the proposed deep architecture for mapping and classifying the scalp data is presented in Section 6, and the experiments are listed in Section 7. Section 8 concludes our work on the mapping and classification of sEEG.

\section{Problem Formulation}

The problem of mapping scalp to intracranial EEG can be posed as a source separation problem. Consider the sEEG signals $\mathbf{x}(n)$, where each observed signal is comprised of a number of unobservable source signals (i.e intracranial EEG) $\mathbf{s}(n)$, and their relationship can be modeled as

$$
\mathbf{x}_{N}(n)=\mathbf{H}_{N} \mathbf{s}_{N}(n)
$$

where $\mathbf{H}$ and the subscript ' $N$ ' denote the unavailable mapping matrix model of size $(\tau \times m)$ to be estimated and the $N$ th subject respectively. In the context of our problem, $\tau>m$, as the number of sEEG channels exceeds the number of intracranial EEG channels. The scalp data is treated as a noisy version of the intracranial data and the goal here is to "reduce" the noise by estimating the iEEG from the sEEG. Moreover, the patient of interest whose brain mapping and iEEG are unknown, is always considered to be the last subject in a population of $N$ subjects. The problem is twofold. First, the inverse of mapping model $\mathbf{H}_{N}$ (i.e. $\mathbf{W}_{N}$ ) needs to be estimated to reconstruct the intracranial EEG $\mathbf{s}_{N}(n)$. Second, the estimated intracranial EEG $\hat{\mathbf{s}}_{N}(n)$ needs to be labeled as either IED or non IED, which can be expressed as

$$
P_{N}=g\left(\hat{\mathbf{s}}_{N}(n)\right)
$$

where $P_{N}$ is the classification accuracy for subject $N, g()$ is the function implemented by a classifier and $\hat{\mathbf{s}}_{N}(n)$ is the estimated pseudo-iEEG generated by the mapping model.

In contrast to blind source separation, here we do not need to make assumptions that may not be realistic in practice. For example, the statistical indepen- 


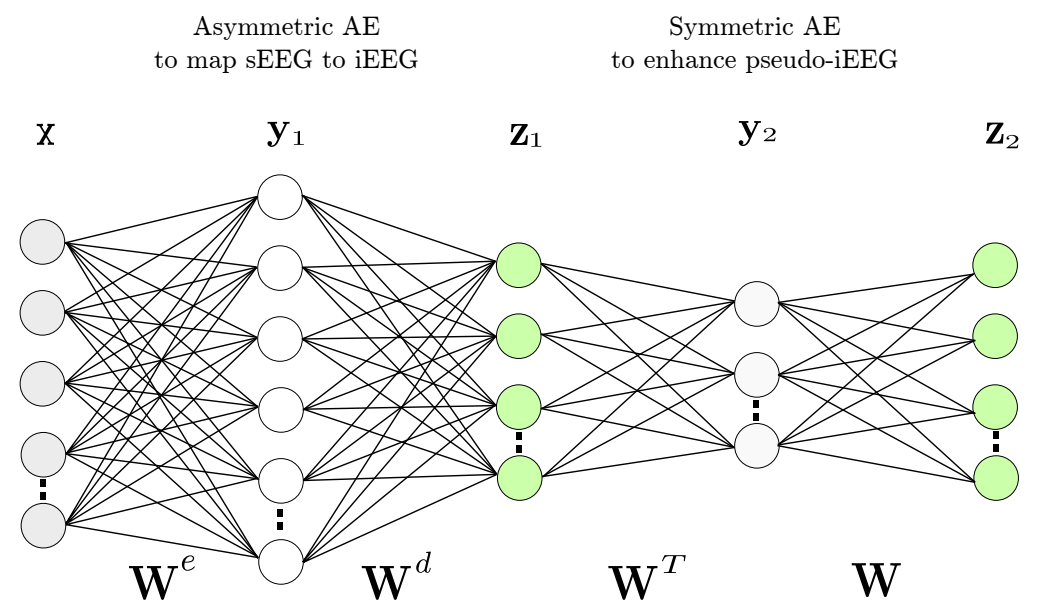

Figure 2. Topology of the Asymmetric-Symmetric Autoencoder (ASAE). $\mathbf{x}$ is the sEEG, $\mathbf{y}_{1}$ is the hidden layer of the Asymmetric Auto-Encoder (AAE), $\mathbf{z}_{1}, \mathbf{z}_{2}$ are the estimated sources of iEEG, $\mathbf{y}_{2}$ is the hidden layer of the Autoencoder $(\mathrm{AE}), \mathbf{W}^{e}$ and $\mathbf{W}^{d}$ are the weights of the AAE and $\mathbf{W}$ are the tied weights of the AE.

dence of the sources may not be an accurate assumption, since they may be correlated due to connectivity within the brain ${ }^{27}$. Instead, we leverage the EEG datasets of the other $N-1$ subjects to approximate the inverse human brain mapping $\mathbf{W}$, where both the sEEG $\mathbf{x}(n)$ and iEEG $\mathbf{s}(n)$ are available. Thus, the inverse mapping $\mathbf{W}$ is more straightforward for each of these $N-1$ subjects than that of the $N$ th patient. Deep learning of neural networks is exploited in two ways. First, autoencoders are modeled to address the estimation problem in Eq. (1), which was not previously considered in our works ${ }^{13,23,34}$. Second, the classification problem Eq. (2) of intracranial EEG is solved by CNNs, whose filters have been designed and optimized for characterizing intracranial epileptic discharges.

\section{Autoencoders}

Autoencoders (AEs), shown in Fig. 2, enable neural networks to train layer by layer in an unsupervised manner. This layer by layer training allows the neural network to break down virtually the complex learning process into simpler and more efficient learning processes across different layers. In other words, the training process in AEs is achieved by greedy layerwise training of multiple layer learning processes, rather than learning from traditional backpropagation in neural networks. As a result, the layers are trained one by one using stochastic gradient descent to minimize the error between the input and the output. When the layers are trained, they are combined as shown in Fig. 2. The unsupervised learning of autoencoders is next explained in the context of IEDs. As illustrated in the right hand side of Fig. 2, the number of (green) input neurons matches the number of (green) output neurons in AEs.

For compact representation of the data, an AE "encodes" an input $\mathbf{x}(n)$ by mapping into a smaller number of neurons $\mathbf{y}(n)$ in the hidden layer of the neural network, such that:

$$
\mathbf{y}(n)=\phi\left(\mathbf{W}^{T} \mathbf{x}(n)+\mathbf{b}\right)
$$

where $\phi(\cdot)$ is the so-called activation function which models the nonlinear mapping, $\mathbf{W}$ is the weight matrix, $\mathbf{b}$ denotes the activation bias, and $(.)^{T}$ is the transpose operator. To verify that the encoded compact representation $\mathbf{y}(n)$ is optimal, $\mathbf{y}(n)$ is decoded (estimated) into the input $\mathbf{x}(n)$ in the form of $\mathbf{z}(n)$ :

$$
\mathbf{z}(n)=\phi(\mathbf{W} \mathbf{y}(n)+\mathbf{b})
$$

Observe that the training does not require any output target; instead, the output $\mathbf{y}(n)$ is mapped back into the input $\mathbf{x}(n)$. This is why it is common to consider the learning process of AEs as unsupervised. To reduce the number of free parameters, the weight matrix $\mathbf{W}$ used in the encoding, see Eq. (3), is reused in the decoding Eq. (4); this process is often referred to as tied weights and has the effect of reducing the number of parameters during training. 


\section{Asymmetric Autoencoders}

As the dimensionalities of iEEG $\mathbf{s}_{j}(n)$ and sEEG $\mathbf{x}_{j}(n)$ are likely to differ, we consider the asymmetric autoencoder (AAE) to determine the mapping from $\mathbf{x}_{j}(n)$ to $\mathbf{s}_{j}(n)$. Unlike AEs, which are symmetric, asymmetric structures require two weight matrices of different dimensions $\mathbf{W}^{e}$ and $\mathbf{W}^{d}$ for the encoding and decoding operations respectively, which can be expressed as:

$$
\begin{aligned}
& \mathbf{y}(n)=\phi\left(\mathbf{W}^{e} \mathbf{x}(n)+\mathbf{b}\right) \\
& \mathbf{z}(n)=\phi\left(\mathbf{W}^{d} \mathbf{y}(n)+\mathbf{b}\right)
\end{aligned}
$$

where $\mathbf{x}$ is the observed signal (sEEG) and $\mathbf{z}$ is the estimated source (iEEG) signal. Compared to AEs, the optimization for asymmetric AEs is more challenging, as it requires two sets of weights $\left\{\mathbf{W}^{e}, \mathbf{W}^{d}\right\}$ to be optimized at each layer instead of one. For such optimization, we have considered the cross-entropy of the reconstruction as the error function.

$$
\begin{aligned}
& L(\mathbf{s}(n), \mathbf{z}(n))= \\
& \quad-\sum_{k=1}^{d}\left[s_{k}(n) \log z_{k}(n)+\left(1-s_{k}(n)\right) \log \left(1-z_{k}(n)\right)\right]
\end{aligned}
$$

where $d$ is the dimensionality of $\mathbf{z}(n)$ and $\mathbf{s}(n)$, and $\log ($.$) is the logarithm function.$

Fig. 2 shows the proposed topology of AEs to map the sEEG onto iEEG. The first stage (left-hand side) is comprised of the asymmetric AE which maps the dimensionality of the sEEG to that of the iEEG. It takes the sEEG, $\mathbf{x}$, as the input and produces the iEEG estimate of a trial in its output. In the second stage (right-hand side), an AE enhances the estimated pseudo-iEEG. This is performed using the pseudo-iEEG estimated by the asymmetric AE, and mapping it to the true iEEG to increase the quality of the estimation. In short, the ASAE architecture takes as input the sEEG, $\mathbf{x}$, of a subject and outputs the estimated iEEG, $\mathbf{z}_{\mathbf{2}}$, for each trial. To address the estimation problem in Eq. (1), the topology of the neural network considered in Fig. 2 is adequate, however AEs only exploit cross-channel information and cannot capture 'local' temporal information within each channel. This property is crucial in the classification of EEG data, since the detection of temporal and morphological bio-markers such as IEDs can assist in the classification process. To this end, CNNs are considered for the classification of the pseudointracranial EEG described in Eq. (2), as explained in the next section.

\section{Convolutional Neural Networks}

Based on the human visual cortex, a CNN has enhanced modeling capability over a traditional neural network, since it can exploit filters that can learn hierarchical representations of the data. To achieve this, the input space is first convolved by each of those filters, then passed through a non-linear function to create a feature map. This feature map represents the useful information in the data. In terms of operation, the $o^{\text {th }}$ filtering and nonlinear process at layer $l$ can be expressed as:

$$
\mathbf{Z}_{i, j, o}^{l}=\phi\left(\sum_{c=1}^{C} \sum_{k=1}^{L}\left(\mathbf{F}_{[i, j, o]}^{l} \mathbf{Z}_{[i+c-1, j+k-1]}^{l-1}\right)+b^{l}\right)
$$

where $L$ is the filter length, $C$ is the number of channels, $\mathbf{F}_{o}^{l}$ is the $o^{t h}$ filter at layer $l, \mathbf{Z}^{l-1}$ is a feature map at layer $l-1$ after applying the hyperbolic tangent non-linear function, $j$ represents the $j^{\text {th }}$ channel and $b^{l}$ is the activation bias for layer $l$.

CNNs have been used traditionally in image processing, which required the filters to be two dimensional. In our work, however, we are interested only in channel-wide information. As such, one dimensional filters are more appropriate to capture only the temporal features. This circumvents the issue of channel mixing. The one dimensional filter convolution has been designed to process a multi-channel signal as:

$$
\mathbf{Z}_{i, j, o}^{l}=\phi\left(\sum_{g=1}^{\Phi^{l-1}} \sum_{k=1}^{L} \mathbf{F}_{j, o}^{l} \mathbf{Z}_{[i, j+k, g]}^{l-1}+\mathbf{b}^{l}\right)
$$

where $j=\{1, . ., C\}, o=\left\{1, . ., \Phi^{l}\right\}$ and $\Phi^{l}$ is the number of filters in the $l^{\text {th }}$ layer.

Following each convolutional layer, a pooling layer is generally utilized to map a window of the generated feature map to a scalar value. Pooling has been proven to achieve invariance to transformation and a more compact representation of images. Although pooling has been proved to be beneficial to deep learning for image processing, it has been found to impede the training of CNNs with epileptic EEG data $^{34}$. This is due to loss of information regarding IED morphology and as a result, pooling has been omitted in this work. 

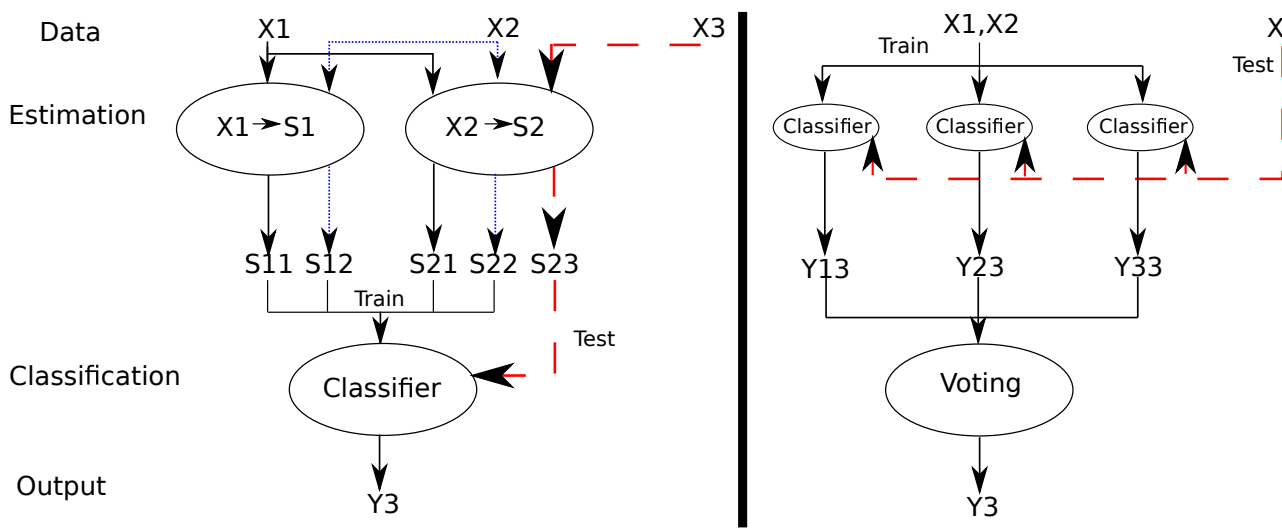

Data

Classification

Figure 3. Visual comparison between our proposed method (left) and an ensemble model (right). In this scenario, consider $X_{2}$ to be more similar to $X_{3}$ than $X_{1}$. On the right side, both $X_{1}$ and $X_{2}$ are used to train the classifiers in order to predict $X_{3}$. Whereas, the left model only uses the estimator trained with $X_{2}$ to generate the unknown signal $S_{3}$. This estimation $\left(S_{23}\right)$ is used to predict the classes of $Y_{3}$.

\section{The proposed deep neural network for the classification of scalp EEG}

For clarity, it is instructive to present our overall proposed method over a numerical example shown in Fig. 3. On its left hand side lies our proposed deep neural architecture to classify sEEG, whereas the right-hand side figure shows the state-of-the-art ensemble ${ }^{29}$ model traditionally used for classification tasks.

Ensemble learning relies upon several models such as neural networks, support vector machines and Bayesian techniques, which train independently or collaboratively on the training data. In our example, the models train on $\left\{\mathbf{x}_{1}, \mathbf{x}_{2}\right\}$. To predict the label for $\mathbf{x}_{3}$, the ensemble either aggregates the results of each model $\left\{\mathbf{y}_{13}, \mathbf{y}_{23}, \mathbf{y}_{33}\right\}$ or selects the best model during training to undertake the classification. This results in the predicted labels $\mathbf{y}_{3}$. To our best knowledge, ensemble learning is the closest methodology to our proposed approach.

In contrast to ensemble learning models, our approach first estimates hidden states $\left\{\mathbf{s}_{11}, \mathbf{s}_{12}, \mathbf{s}_{21}, \mathbf{s}_{22}\right\}$ which exhibit additional features; these features may not always manifest in the data $\left\{\mathbf{x}_{1}, \mathbf{x}_{2}\right\}$, yet provide further insights into the data such as IED. The hidden states which are estimated from autoencoders, are then used to train the CNN.

In the context of our work, $\mathbf{s}_{i j}=\mathbf{W}_{i} \mathbf{x}_{j}$ denotes the estimated intracranial EEG of the $j$ th subject based on the model derived from the $i$ th subject. To classify the unlabeled test sEEG data of subject 3 (i.e. $\mathbf{x}_{3}$ ), only the model (from $\mathbf{x}_{2}$ ) closest to test scalp data $\mathbf{x}_{3}$ is considered to estimate the corresponding intracranial EEG data of the latter (i.e. $\left.\mathbf{s}_{23}\right)$.

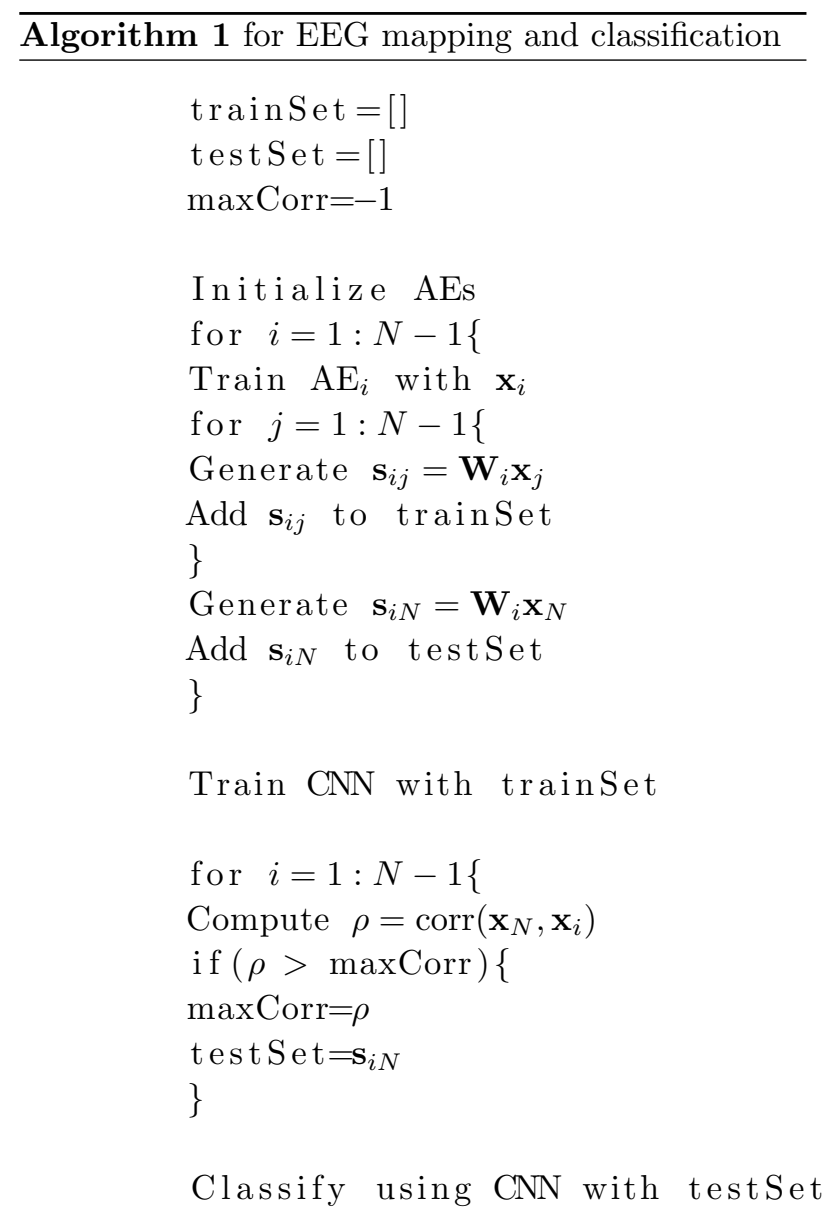


To determine the autoencoder model closest to the test scalp data ( such as $\mathbf{x}_{3}$ ), a simple correlation analysis between the test scalp data and the training scalp data is carried out, since both datasets are available. This simple yet crucial step leads to enhanced estimation of the intracranial EEG of the test data, as demonstrated experimentally in Section 7.1 and analytically in Appendix 1. Our proposed methodology for the mapping and classification of sEEG is summarized in Algorithm 1, where $\operatorname{corr}\left(\mathbf{x}_{N}, \mathbf{x}_{i}\right)$ denotes the Pearson correlation coefficient function between $\mathbf{x}_{N}$ and $\mathbf{x}_{i}$.

\section{Experiments}

The proposed and competing models were built using the python open-source framework Theano ${ }^{30}$ and run on a GTX 980ti.

\subsection{Simulations using synthetic signals}

The objective of this experiment is to demonstrate that the model trained with the signal closest to the signal of interest (test signal) achieves the highest accuracy. To this end, we consider a classification problem of synthetic signals, which are defined as follows:

$$
\begin{aligned}
& x_{1}(n)=\sin \left(n-\frac{\pi}{4}\right)+\varepsilon(n) \\
& x_{2}(n)=\sin (n)+\varepsilon(n) \\
& x_{3}(n)=\frac{1}{2}-\tan ^{-1}\left[\cot \left(\frac{n}{2 \pi}\right)\right]+\varepsilon(n) \\
& x_{4}(n)=\sin \left[\phi_{0}+2 \pi\left(f_{0} n+\frac{k}{2} n^{2}\right)\right]+\varepsilon(n) \\
& x_{5}(n)=\sin \left[\phi_{0}+2 \pi\left(f_{0}\left(n-\frac{\pi}{4}\right)+\frac{k}{2}\left(n-\frac{\pi}{4}\right)^{2}\right)\right]+\varepsilon(n)
\end{aligned}
$$

where $\varepsilon$ is some random white Gaussian noise with zero mean and standard deviation $=1, \mathbf{x}_{1}(n)$ and $\mathbf{x}_{2}(n)$ are sinusoidal functions, $\mathbf{x}_{3}(n)$ is a sawtooth function, and $\mathbf{x}_{4}(n)$ and $\mathbf{x}_{5}(n)$ are chirp signals. For $\mathbf{x}_{4}(n)$ and $\mathbf{x}_{5}(n)$ the instantaneous frequency $f_{0}$ is 0.1 and the initial phase $\phi_{0}$ is 0 .

Recall that the aim of this experiment is to show that the model trained with the signal closest to the (test) signal of interest attains the highest accuracy. To this end, consider $x_{5}(n)$ as the training data, and the problem is to classify the remaining signals $\left\{x_{1}(n), x_{2}(n), x_{3}(n), x_{4}(n)\right\}$.
A comprehensive set of simulations were carried out over a range of SNR and the results are summarized in Fig. 4. It is clear from Eq. (10) that $x_{5}(n)$ is most similar to $x_{4}(n)$, and most dissimilar to $x_{3}(n)$. This is why the model provided the highest classification accuracy for $x_{4}(n)$ whereas the lowest accuracy was obtained for the classification of $x_{3}(n)$. For rigor, we also show analytically in Appendix 1 why a model trained with data closest to the data of interest provides superior performance. Now that the rationale underlying Algorithm 1 for mapping and classification of sEEG has been established, we proceed with assessing the efficacy of our proposed method (shown in Fig. 3) in a real-world application: detection of intracranial epileptic discharges from sEEG.

\subsection{Detection of IEDs from scalp EEG}

\subsubsection{Dataset}

The datasets of the epileptic subjects ${ }^{13}$ are summarized in Table 1, which includes a total of 18 subjects; 11 males and 7 females. A number of trials were examined for each subject to allow for a dataset large enough to have statistical significance. Each subject was assessed for temporal lobe epilepsy using 12 FO and 20 scalp electrodes at King's College Hospital London. Each trial lasted $325 \mathrm{~ms}$ and was digitized at $200 \mathrm{~Hz}$. As the number of electrodes and therefore the channels, differed between scalp and intracranial recordings, each trial resulted in a dataframe of size [time $(325) \times$ frequency $(0.2) \times$ No. of channels]. In essence, the data for each subject had a size of $[1300 \times$ No. of trials $]$ for the sEEG and $[780 \times$ No. of trials] for the iEEG.

Table 1. Summary of scored data.

\begin{tabular}{c|c|c|c}
\hline Subject & No. of trials & $\underline{\text { Subject }}$ & No. of trials \\
\hline S1 & 684 & S10 & 448 \\
S2 & 100 & S11 & 1696 \\
S3 & 144 & S12 & 1906 \\
S4 & 330 & S13 & 1658 \\
S5 & 316 & S14 & 1082 \\
S6 & 944 & S15 & 520 \\
S7 & 398 & S16 & 1212 \\
S8 & 634 & S17 & 228 \\
S9 & 682 & S18 & 236 \\
\hline
\end{tabular}

The data was scored by an expert epileptologist into the IED positive and non-IED classes. Examining 


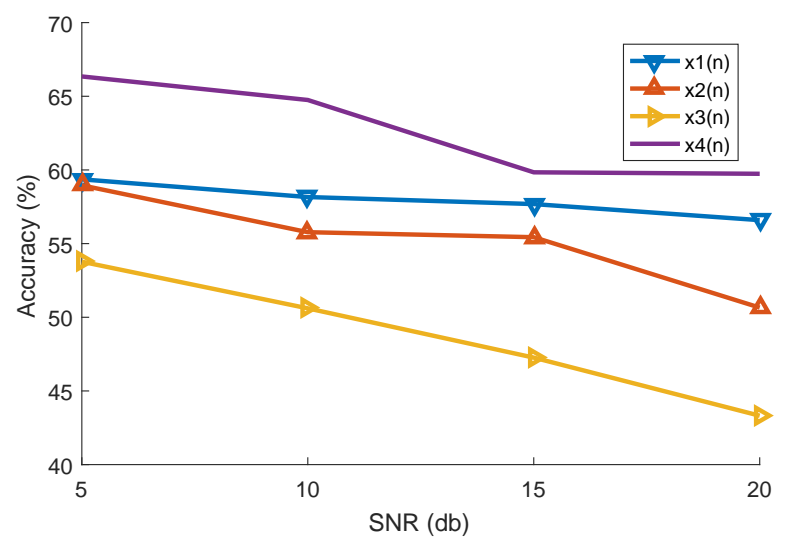

Figure 4. Classification of $x_{1}(n), \ldots, x_{4}(n)$ defined in Eq. (10) based on a model trained on $x_{5}(n)$ at different noise levels.
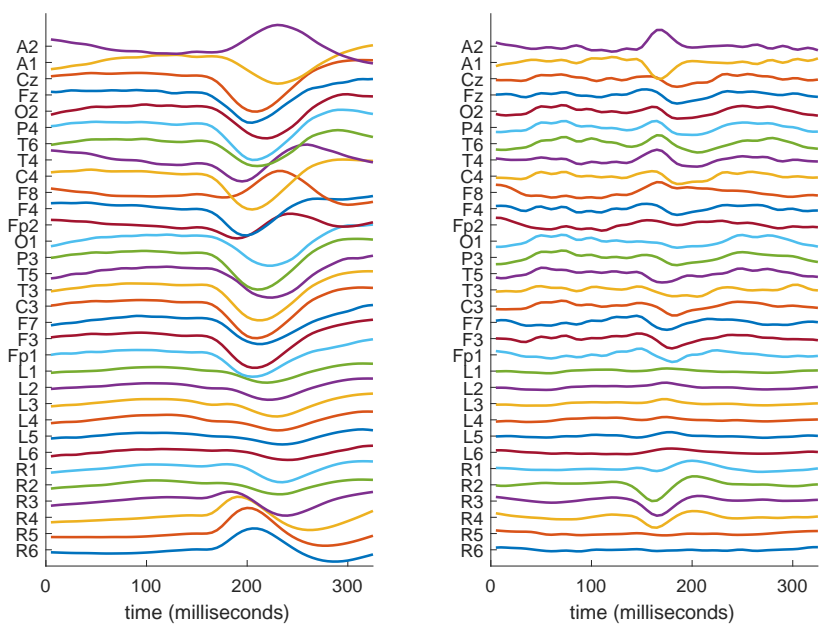

Figure 5. Waveform difference in IED segments for two subjects.

Fig. 5 and Fig. 6, observe the variability between subjects in terms of the IED and non-IED trials respectively. The vast variations in the waveforms make the accurate detection of IEDs from EEG data a challenging machine learning problem.

\subsubsection{Experimental Setup}

To address this high degree of variability, we adopted the leave-subject-out method; our proposed deep AEs were trained using the data from 17 subjects to map the sEEG to iEEG for each, using the sEEG as the input and the iEEG as the target. Each of the 17 autoencoders (AAE and ASAE) was used to create an estimation of each of the other subjects' intracranial data. To enable this, the autoen- coders were trained using the sEEG and iEEG of the 17 subjects, one subject per AE. The estimated pseudo-iEEG were used as input to the CNN and were divided into two proportional sets, validation and training. To generate the test data the sEEG of the 18th subject (test subject) was used. The cross correlations between the test subject's sEEG and the other 17 subjects were computed. The AE trained with the subject that had the highest correlation to the test subject was used to estimate the test pseudo-iEEG. Finally, the pseudo-iEEG of the test subject was passed as input to the CNN for feature extraction and classification. For completeness, we have also performed within-subject experiments where only half the data of a subject was used as the training set and the remaining half was used as the 

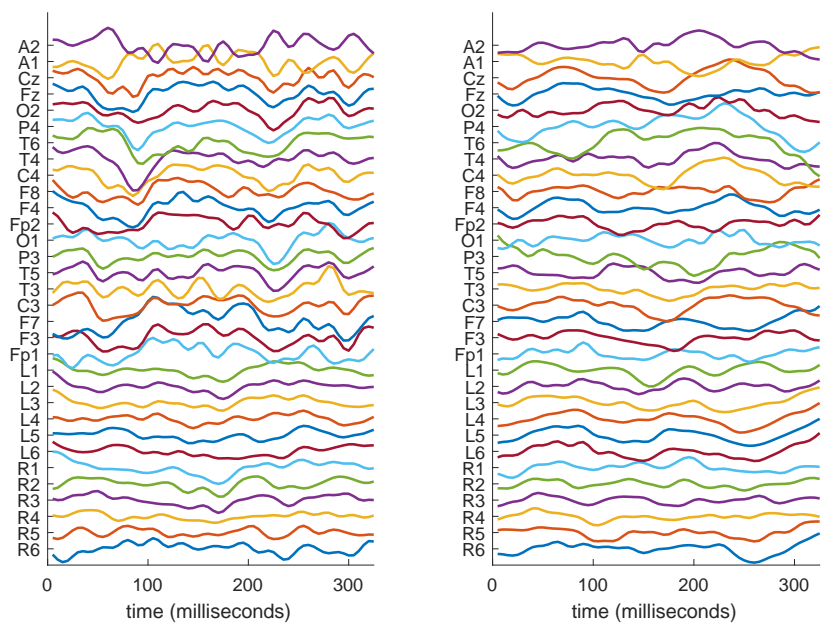

Figure 6. Waveform difference in normal brain activity (non-IED) segments for two subjects.

testing set.

\subsubsection{Competing models}

A number of models have been proposed for the analysis of EEG data for epilepsy. These include continuous wavelet transform ${ }^{31}$, chirplet transform ${ }^{32}$ and time domain data. In a recent work by Spyrou et al. ${ }^{13}$, it was discovered that time-frequency (TF) features were superior to the above methods in this dataset. As a result, the TF features ${ }^{13}$ were considered as the state-of-the-art. Additionally, the linear mapping proposed by Kaur et. $\mathrm{al}^{14}$ is used as it considered a similar mapping to the proposed methodology. Finally, a benchmark AE model was considered as a third comparison to the proposed models. The three competing methodologies compared against the proposed asymmetric autoencoder (AAE) and asymmetric symmetric autoencoder (ASAE) are as follows:

- $\underline{\text { TF}}$ : A recent TF approach ${ }^{13}$ for feature extraction from the sEEG data and a logistic regression classifier.

- Linear Regression: A linear regression model for mapping sEEG to iEEG and a classifier trained with stepwise discriminant analysis ${ }^{14}$.

- $\underline{\mathrm{AE}}$ : A benchmark deep learning $\mathrm{AE}^{28}$ for feature extraction from the sEEG data and a logistic regres- sion classifier.

For fair comparisons, the AE models were set approximately to the same number of parameters shown in Table 2. All models trained on the same dataset. The input neurons of the AEs reflect the dimensionality of the sEEG data, which were recorded with 20 electrodes, as described in Section 7.2.1. The activations of the AAE and ASAE were passed to a CNN for feature extraction. All 5 models were followed by a logistic regressor with the cross-entropy loss function in Eq. (7) and trained with stochastic gradient descent. For the CNN model, the same architecture and training procedure were used as in our previous work $\mathbf{3 4}^{\mathbf{3 4}}$. The input layer of the CNN consisted of 780 neurons, as this reflected the dimensionality of the iEEG and pseudo-iEEG data as discussed in Section 7.2.1. After initial experimentation and finetuning of parameters, the optimal CNN had 4 convolutional layers, a fully connected hidden layer, and a softmax layer for the classification. For the convolutional layers, the first filter size was set to $32(160 \mathrm{~ms})$ since this duration was adequate to capture the main part of IED waveforms. The filters for the following convolutional layer were gradually reduced to capture finer details of the $\operatorname{IEDs}^{33}$.

\subsubsection{Results and Discussions}

Fig. 7 shows the estimated iEEG (second and third row) mapped from sEEG (first row). It is clear that 
Table 2. Training parameters for Autoencoders

\begin{tabular}{l|c|c|c}
\hline Parameter & AE & AAE & ASAE \\
\hline Input neurons & 1300 & 1300 & 1300 \\
Hidden layers & 5 & 1 & 2 \\
Hidden neurons & $1500,780,500,100,50$ & 1500 & 1500,500 \\
Activation function & Hyberbolic tangent & Hyberbolic tangent & Hyberbolic tangent \\
\hline Network parameters & $4.06 \times 10^{6}$ & $3.12 \times 10^{6}$ & $3.90 \times 10^{6}$ \\
Output features & 50 & 780 & 780 \\
\hline
\end{tabular}
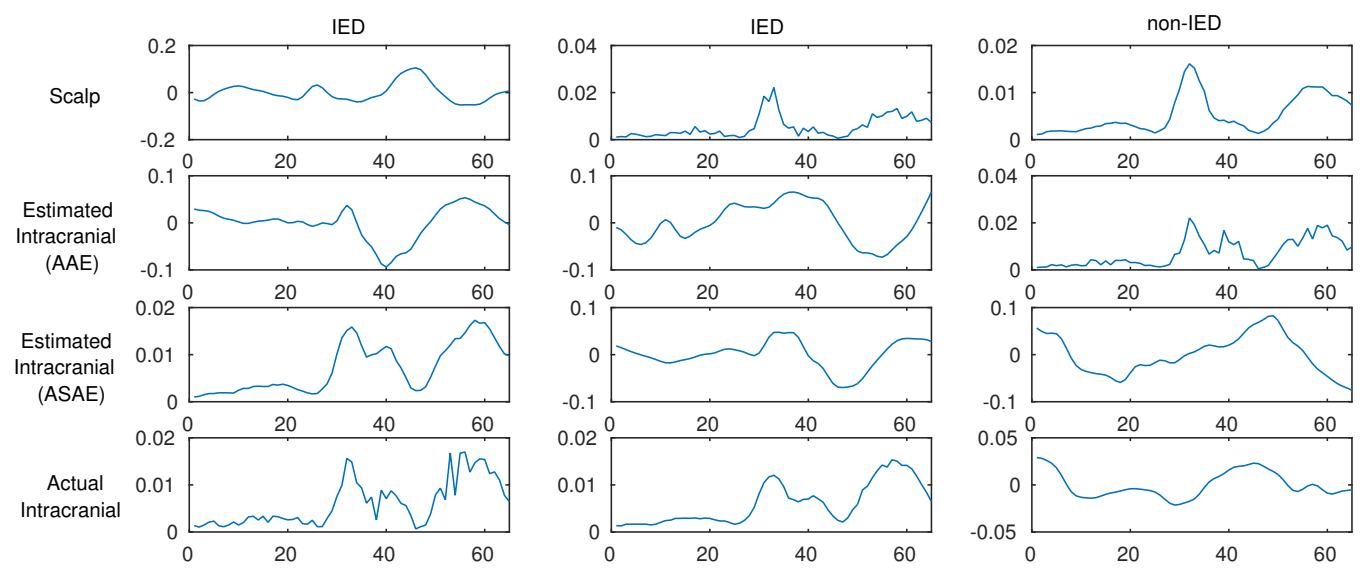

Figure 7. Estimation of iEEG for two IED segments and a non-IED segment (averaged over all channels) using AAE and ASAE. In the case of ASAE, the additional symmetric layer led to a smoother estimation of the intracranial data.
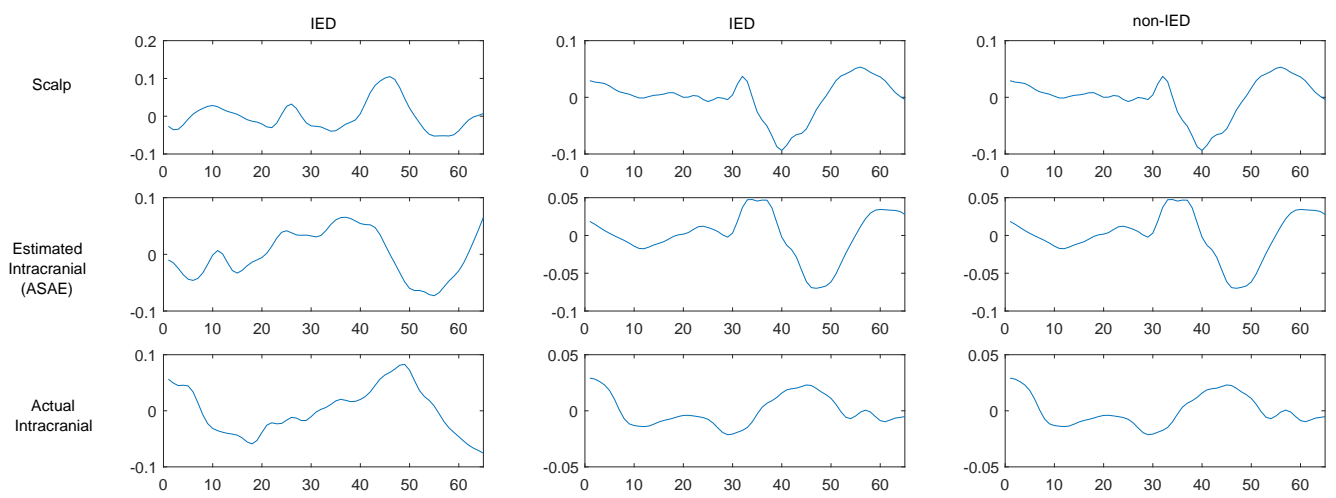

Figure 8. Estimation of iEEG for two IED segments and a non-IED segment (averaged over all channels) using AAE and ASAE. In the case of ASAE, the additional symmetric layer led to a smoother estimation of the intracranial data.

the proposed ASAE provided better estimates than those by the proposed AAE. The deeper learning architecture of ASAE led to this superior mapping performance, indicating that ASAE is likely to have better classification accuracy than AAE. Table 3 confirms that it is indeed the case, and also compares against recent competing techniques ${ }^{13,28}$. As Fig. 7 offers a qualitative assessment of the mapping, we have provided a more quantitative measure of success through testing the no correlation hypothesis for all the subjects, see Table 5 . For testing the statistical significance between the different methods, we used the binomial confidence interval. In order to assess for significant differences between the two methods, we calculated the confidence interval for one and checked whether the accuracy of the 
Table 3. Classification accuracy per subject for different approaches.

\begin{tabular}{|c|c|c|c|c|c|}
\hline Subject & $\mathrm{AE}^{28}$ & Linear Regression $^{14}$ & $\mathrm{TF}^{13}$ & AAE-CNN & ASAE-CNN \\
\hline 1 & $56(60)$ & $65(72)$ & $71(78)$ & $85(80)$ & $87(78)$ \\
\hline 2 & $67(70)$ & $86(81)$ & $81(75)$ & $92(82)$ & $94(88)$ \\
\hline 3 & $58(67)$ & $65(69)$ & $68(75)$ & $72(72)$ & $69(82)$ \\
\hline 4 & $55(58)$ & $58(62)$ & $58(65)$ & $58(71)$ & $59(77)$ \\
\hline 5 & $56(56)$ & $55(55)$ & $57(57)$ & $64(64)$ & $65(75)$ \\
\hline 6 & $57(57)$ & $61(59)$ & $73(75)$ & $71(60)$ & $71(63)$ \\
\hline 7 & $54(56)$ & $59(64)$ & $60(64)$ & $54(62)$ & $67(72)$ \\
\hline 8 & $57(57)$ & $55(66)$ & $58(64)$ & $55(62)$ & $57(68)$ \\
\hline 9 & $54(63)$ & $63(65)$ & $70(72)$ & $61(74)$ & $62(68)$ \\
\hline 10 & $52(63)$ & $66(70)$ & $75(78)$ & $71(65)$ & $74(77)$ \\
\hline 11 & $55(54)$ & $63(64)$ & $61(63)$ & $65(67)$ & $65(68)$ \\
\hline 12 & $69(77)$ & $73(79)$ & $67(71)$ & $75(84)$ & $77(84)$ \\
\hline 13 & $55(58)$ & $62(71)$ & $66(72)$ & $62(72)$ & $64(71)$ \\
\hline 14 & $55(55)$ & $59(62)$ & $62(65)$ & $66(71)$ & $67(65)$ \\
\hline 15 & $52(50)$ & $50(46)$ & $44(54)$ & $50(53)$ & $50(52)$ \\
\hline 16 & $66(64)$ & $51(55)$ & $61(62)$ & $67(77)$ & $68(72)$ \\
\hline 17 & $56(67)$ & $54(62)$ & $66(74)$ & $59(54)$ & $62(71)$ \\
\hline 18 & $66(64)$ & $66(64)$ & $57(50)$ & $61(53)$ & $67(75)$ \\
\hline Mean & $58(61)$ & $62(65)$ & $65(67)$ & $66(68)$ & $68(73)$ \\
\hline
\end{tabular}

The accuracy of the leave-subject-out method in terms of classifying the IED and non-IED segments. The numbers in brackets denote the within-subject accuracy.

second method exceeds that interval. These confidence intervals indicate the range of classification accuracies that can arise by chance (within a $95 \%$ condifence level). In the case of Linear Regression and ASAE-CNN, the subject independent performance exceeded the confidence interval for all the subjects except S8, S15 and S18. For the comparison between TF and ASAE-CNN, the subjects whose performances did not exceed the confidence interval were S3, S4, S9, S11 and S17. The following remarks can be made with respect to the results.

\#Remark 1: The deep learning AE performed the worst out of all competing models. This can be attributed to the fact that unlike the other competing models, the AE was used for feature extraction from sEEG. Deep learning techniques can be used in many areas and have exceeded the state-of-the-art in many applications. To this end, the particularities of the data need to be taken into account. In this work, the consideration of asymmetric $\mathrm{AEs}$ and 1-d CNNs for feature extraction was a crucial factor for the improved performance.

\#Remark 2: The highest accuracy increase was observed in within-subject experiments as expected, where the proposed ASAE method outperformed those trained with TF and AAE features by $6 \%$ and $5 \%$ respectively.

\#Remark 3: For subjects 1 and 2, the withinsubject accuracy is worse than the subject independent accuracy. This means that training on data from other subjects has benefited our proposed models more than training on their own data.

\#Remark 4: The ROC curves in Fig. 9 re-affirms the improved accuracy of our proposed ASAE. Table 3 indicated that TF and the proposed AAE had almost identical performances, yet Fig. 9 demonstrates the superior performance of the proposed AAE method due to the more in depth analysis entailed by the ROC.

\#Remark 5: For rigor, a statistical analysis for the performance of each method is also provided in Table 4. Observe that the ASAE-CNN approach has superior performance in terms of True Positives, confirming that the additional non-linear layer is beneficial to detecting IEDs, as suggested by Fig. 7 .

\#Remark 6: The hypothesis of no correlation was 
tested between the estimated pseudo-iEEG and the true iEEG as a statistical verification. The results showed that the no correlation hypothesis was rejected for all the subjects as shown in Table 5 . This further confirms that the estimated pseudointracranial had high fidelity with the true iEEG data.

\#Remark 7: Although both AAE-CNN and ASAECNN architectures have outperformed the competing models, the computational complexity involved in training such deep networks must be considered. As opposed to the TF features and linear regression, deep learning models are time-consuming to train. Even with the use of high end graphics cards, the time required to train such models cannot be emphasized enough. Even so, after a deep model is trained, predictions on unseen data can be done approximately in real-time.

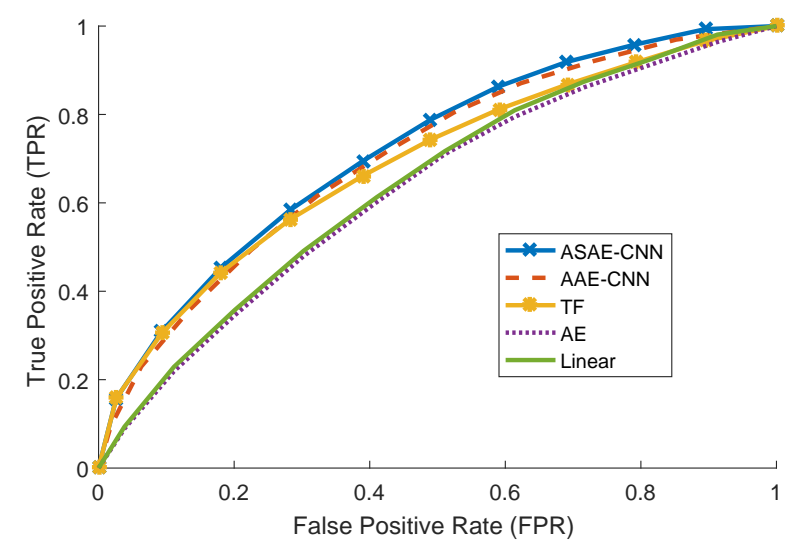

Figure 9. ROC curves for the competing methods: Areas under the curves are 0.63(AE), 0.64(Linear Regression), 0.67(TF), 0.72(AAE), and 0.74(ASAE).

\section{Conclusion}

Deep learning can benefit clinical neuroscience through automatic feature generation and improved classification accuracy, given enough computational processing power. In this work, we have proposed an ensemble deep learning architecture for non-linearly mapping scalp to iEEG. In order to accurately detect epileptic discharges from sEEG of a subject, we have leveraged the iEEG information of other subjects. To this end, asymmetric autoencoders have been introduced and used to map the sEEG to the iEEG and circumvent the issue of dimensionality difference.

The proposed methodology offers a novel way to estimate iEEG, taking a step towards circumventing the need to undertake invasive surgical intervention. To enable the deep architecture to better estimate the iEEG of an unseen subject, we have used the mapping model trained on the subject with the highest correlated sEEG data to the test subject. Using the estimated iEEG data from this model, our classifiers were able to outperform the state-of-the-art in classification accuracy. For statistical rigor, the estimated pseudo-intracranial EEG were compared with the true iEEG and were found comparable as the 'no correlation' hypothesis was rejected.

Future work includes the use of deep subdural electrodes as an alternative source of iEEG data as well as deeper computational models to enhance the mapping performance of the proposed architecture. Additionally, extending the study group to subjects with epileptic focus on extratemporal locations could help generalize the proposed approach.

\section{Appendix A}

The aim is to provide a sketch demonstration that the estimate of source $\mathbf{s}_{N}(n)$ based on a model trained on data $\mathbf{x}_{j}(n)$ is the best estimate, given that the expectation $\mathrm{E}\left\{\mathbf{s}_{N}(n) \mathbf{s}_{j}(n)\right\}>\mathrm{E}\left\{\mathbf{s}_{N}(n) \mathbf{s}_{i}(n)\right\}$, where $i \neq j \neq N$.

Consider two uncorrelated variables $\mathbf{s}_{1}(n), \mathbf{s}_{2}(n)$ such that $\mathrm{E}\left\{\mathbf{s}_{1}(n) \mathbf{s}_{2}(n)\right\}=0$. These can be used to generate a generic $\mathbf{s}_{k}(n)$ signal such that:

$$
\mathbf{s}_{k}(n)=\rho \mathbf{s}_{1}(n)+\mathbf{s}_{2}(n) \sqrt{1-\rho^{2}}
$$

for $0 \leq \rho \leq 1$. Consider $\mathbf{s}_{N}(n)=\mathbf{s}_{1}(n)$, which is the unknown source of its noisy version $\mathbf{x}_{N}(n)$ and substitute Eq. (A.1) to derive the correlation between $\mathbf{s}_{N}(n)$ and an arbitrary $\mathbf{s}_{k}(n)$ as:

$$
\begin{aligned}
\mathrm{E}\left\{\mathbf{s}_{N}(n) \mathbf{s}_{k}(n)\right\} & =\mathrm{E}\left\{\mathbf{s}_{1}(n)\left[\rho \mathbf{s}_{1}+\mathbf{s}_{2}(n) \sqrt{1-\rho^{2}}\right]\right\} \\
& =\mathrm{E}\left\{\mathbf{s}_{N}(n)\left[\rho \mathbf{s}_{N}(n)+\mathbf{s}_{2}(n) \sqrt{1-\rho^{2}}\right]\right\}
\end{aligned}
$$

To model a non-correlated and a correlated signal to $\mathbf{s}_{N}(n)$ we can adapt $\rho$ in Eq. (A.2) to have:

$$
\begin{aligned}
\rho=0 & \Longrightarrow \mathbf{s}_{i}(n)=\mathbf{s}_{k}(n)=\mathbf{s}_{2}(n) \\
& \Longrightarrow \mathrm{E}\left\{\mathbf{s}_{N}(n) \mathbf{s}_{i}(n)\right\}=0 \\
\rho=1 & \Longrightarrow \mathbf{s}_{j}(n)=\mathbf{s}_{k}(n)=\mathbf{s}_{N}(n) \\
& \Longrightarrow \mathrm{E}\left\{\mathbf{s}_{N}(n) \mathbf{s}_{j}(n)\right)=\mathrm{E}\left\{\mathbf{s}_{N}^{2}(n)\right\}
\end{aligned}
$$


Table 4. Statistical results for different approaches.

\begin{tabular}{l|c|c|c|c|c|c|c|c}
\hline Method & TP & FP & FN & TN & Precision & Sensitivity & f-measure & Specificity \\
\hline AE & 7745 & 5473 & 6123 & 7095 & 0.59 & 0.56 & 0.57 & 0.56 \\
Linear Regression & 7553 & 5665 & 4897 & 8321 & 0.57 & 0.61 & 0.59 & 0.59 \\
TF & 8214 & 5004 & 4456 & 8762 & 0.62 & 0.65 & 0.63 & 0.64 \\
AAE-CNN & 8369 & 4849 & 4273 & 8945 & 0.63 & 0.66 & 0.65 & 0.65 \\
ASAE-CNN & 9246 & 3972 & 4591 & 8627 & 0.70 & 0.67 & 0.68 & 0.68 \\
\hline
\end{tabular}

Table 5. Statistical test of no correlation between estimated pseudo-in-

\begin{tabular}{|c|c|c|}
\hline Subject & $\begin{array}{c}\mathrm{p} \text {-value } \\
\text { (confidence threshold 95\%) }\end{array}$ & No Correlation Hypothesis \\
\hline 1 & 0.0216 & Rejected \\
\hline 2 & 0.0297 & Rejected \\
\hline 3 & 0.0491 & Rejected \\
\hline 4 & 0.0472 & Rejected \\
\hline 5 & 0.0383 & Rejected \\
\hline 6 & 0.0175 & Rejected \\
\hline 7 & 0.0283 & Rejected \\
\hline 8 & 0.0190 & Rejected \\
\hline 9 & 0.0241 & Rejected \\
\hline 10 & 0.0308 & Rejected \\
\hline 11 & 0.0107 & Rejected \\
\hline 12 & 0.0198 & Rejected \\
\hline 13 & 0.0102 & Rejected \\
\hline 14 & 0.0104 & Rejected \\
\hline 15 & 0.0183 & Rejected \\
\hline 16 & 0.0178 & Rejected \\
\hline 17 & 0.0447 & Rejected \\
\hline 18 & 0.0256 & Rejected \\
\hline
\end{tabular}

Clearly, when $\rho=1$ the expectation is maximized. Additionally, when $\rho=1$ we have:

$$
\begin{gathered}
\mathbf{s}_{N}(n)=\mathbf{s}_{j}(n)=\mathbf{W}_{j} \mathbf{x}_{j}(n)=\mathbf{W}_{N} \mathbf{x}_{N}(n) \\
\Downarrow \\
\mathrm{E}\left\{\mathbf{s}_{N}(n)\right\}=\mathbf{W}_{N} \mathrm{E}\left\{\mathbf{x}_{N}(n)\right\} \\
\Downarrow \\
\begin{aligned}
\mathrm{E}\left\{\mathbf{s}_{N}(n) \mathbf{s}_{j}(n)\right\}=\mathbf{W}_{N} \mathrm{E}\left\{\mathbf{x}_{N}(n) \mathbf{x}_{j}(n)\right\} \mathbf{W}_{j} \\
=\mathbf{W}_{N} \mathrm{E}\left\{\mathbf{x}_{N}(n) \mathbf{x}_{N}(n)\right\} \mathbf{W}_{N}
\end{aligned}
\end{gathered}
$$

Therefore we have:

$$
\begin{aligned}
\rho \rightarrow 0 & \Longrightarrow \mathbf{s}_{i}(n) \rightarrow \mathbf{s}_{2}(n) \\
& \Longrightarrow \mathbf{x}_{N}(n) \nrightarrow \mathbf{x}_{i}(n), \mathbf{W}_{N} \nrightarrow \mathbf{W}_{i} \\
\rho \rightarrow 1 & \Longrightarrow \mathbf{s}_{j}(n) \rightarrow \mathbf{s}_{N}(n) \\
& \Longrightarrow \mathbf{x}_{N}(n) \rightarrow \mathbf{x}_{j}(n), W_{N} \rightarrow W_{j}
\end{aligned}
$$

Based on Eq. (2), we assume that the highest achievable accuracy is $P_{N}$. We need to show that:

$$
\left|P_{N}-P_{j}\right|<\left|P_{N}-P_{i}\right|
$$

First, we expand $P_{N}, P_{j}$ and $P_{i}$ using Eq. (2):

$$
\begin{aligned}
P_{N} & =g\left(\mathbf{W}_{N} \mathbf{x}_{N}(n)\right) \\
P_{j} & =g\left(\mathbf{W}_{j} \mathbf{x}_{j}(n)\right) \\
P_{i} & =g\left(\mathbf{W}_{i} \mathbf{x}_{i}(n)\right)
\end{aligned}
$$

Second, we substitute Eq. (A.9) in Eq. (A.8) to obtain:

$\left|g\left(\left[\mathbf{W}_{N}-\mathbf{W}_{j}\right] \mathbf{x}_{N}(n)\right)\right|<\left|g\left(\left[\mathbf{W}_{N} \mathbf{x}_{N}(n)-\mathbf{W}_{i} \mathbf{x}_{i}(n)\right]\right)\right|$

Therefore, as $\rho \rightarrow 1, \mathbf{x}_{N}-\mathbf{x}_{j} \rightarrow 0, \mathbf{W}_{N}-\mathbf{W}_{j} \rightarrow 0$. As a result, the absolute difference in performance between the optimal $P_{N}$ and $P_{j}$ is smaller than any other $P_{i}$.

If the expectation of $\mathbf{x}_{N}(n)$ and $\mathbf{x}_{j}(n)$ is higher than 
the expectation of $\mathbf{x}_{N}(n)$ and any other $\mathbf{x}_{j}(n)$ :

$$
E\left\{\mathbf{x}_{N}(n) \mathbf{x}_{j}(n)\right\}>E\left\{\mathbf{x}_{N}(n) \mathbf{x}_{i}(n)\right\}
$$

then as $\rho \rightarrow 1$ and $P_{j} \rightarrow P_{N}$ we have:

$$
\left.\begin{array}{l}
\mathbf{x}_{j}(n) \rightarrow \mathbf{x}_{N}(n) \\
\mathbf{W}_{j} \rightarrow \mathbf{W}_{N}
\end{array}\right\} \Longrightarrow\left|P_{j}-P_{N}\right|<\left|P_{i}-P_{N}\right|
$$

This shows that if the two signals $\mathbf{x}_{N}(n)$ and $\mathbf{x}_{j}(n)$ are more correlated than $\mathbf{x}_{N}(n)$ and $\mathbf{x}_{i}(n)$, then the estimated unknown source $\hat{\mathbf{s}}_{N j}(n)$ is closer to $\mathbf{s}_{N}(n)$ than $\mathbf{s}_{N i}(n)$. As a result, the classification accuracy $P_{j}$ is higher than $P_{i}$.

\section{Bibliography}

1. S. Sanei, 2013, "Adaptive Processing of Brain Signals", 1st ed. John Wiley and Sons.

2. W. Y. Hsu, 2011, "Continuous EEG signal analysis for asynchronous BCI application", International Journal of Neural Systems, vol. 21, pp. 335-350.

3. Q. Yuan, W. Zhou and S. Yuan, 2014, "Epileptic EEG classification based on kernel sparse representation", International Journal of Neural Systems, vol. 24, no. 4.

4. U. R. Acharya, S. V. Sree and J. S. Suri, 2011, "Automatic detection of epileptic EEG signals using higher order cumulant features", International Journal of Neural Systems, vol. 21, pp. 403-414.

5. S. Noachtar, C. Binnie, J. Ebersole, F. Mauguiere, A. Sakamoto and B. Westmoreland, 1999, "A glossary of terms most commonly used by clinical electroencephalographers and proposal for the report form for the EEG findings. The International Federation of Clinical Neurophysiology", Electroencephalogr. Clin. Neurophysiol. Suppl., vol. 52, pp. 21-41.

6. W. Wang, J. L. Collinger, M. Perez, E. C. TylerKabara, L. G. Cohen, N. Birbaumer, S. W. Brose, A. B. Schwartz, M. L. Boninger and D. J. Weber, 2010, "Neural interface technology for rehabilitation: Exploiting and promoting neuroplasticity", Phys. Med. Rehabil. Clin. North Amer., vol. 21, no. 1, pp. 157178.

7. G. Alarcon, C. N. Guy, C. D. Binnie, S. R. Walker, R. D. Elwes and C. E. Polkey, 1994, "Intracerebral propagation of interictal activity in partial epilepsy: implications for source localisation", Journal of Neurology, Neurosurgery and Psychiatry 57, pp. 435-449.

8. N. Kissani, G. Alarcon, M. Dad, C. Binnie and C. Polkey, 2001, "Sensitivity of recordings at sphenoidal electrode site for detecting seizure onset: evidence from scalp, superficial and deep foramen ovale recordings", Clinical Neurophysiology 112, pp. 232-240.
9. S. S. Lodder and M. J. A. M. V. Putten, 2014, "A self-adapting system for the automated detection of inter-ictal epileptiform discharges," PLoS ONE, vol. 9 , no. 1 .

10. F. Grouiller, R. C. Thornton, K. Groening, L. Spinelli, J. S. Duncan, K. Schaller, M. Siniatchkin, L. Lemieux, M. Seeck, C. M. Michel, et al., 2011, "With or without spikes: localization of focal epileptic activity by simultaneous electroencephalography and functional magnetic resonance imaging", Brain, vol. 134, no. 10, pp. 2867-2886.

11. N. Gaspard, R. Alkawadri and P. Farooque, 2014, "Automatic detection of prominent interictal spikes in intracranial EEG: Validation of an algorithm and relationsip to the seizure onset zone", Journal of Neuroscience Methods, vol. 125, pp. 1095-1103.

12. R. Janca, P. Jezdik, R. Cmejla et al., 2015, "Detection of interictal epileptiform discharges using signal envelope distribution modelling: application to epileptic and non-epileptic intracranial recordings", Brain topography, vol. 28.1, pp. 172-183.

13. L. Spyrou, D. Martin-Lopez, A. Valentin, G. Alarcon and S. Sanei, 2016, "Detection of intracranial signatures of interictal epileptiform discharges from concurrent scalp EEG", International Journal of Neural Systems, vol. 26, no. 04, p. 1650016.

14. K. Kaur, J. Shih and D. Krusienski, 2014, "Empirical models of scalp-EEG responses using non-concurrent intracranial responses", Journal of Neural Engineering, vol 11:035012.

15. L. Spyrou and S. Sanei, 2016, "Coupled dictionary learning for multimodal data: An application to concurrent intracranial and scalp eeg", IEEE International Conference on Acoustics, Speech and Signal Processing (ICASSP), pp. 2349-2353.

16. S. Ji, W. Xu, M. Yang and K. Yu, 2013, "3D convolutional neural networks for human action recognition", IEEE Trans. Pattern Analysis and Machine Intelligence, vol.35, no.1, pp.221-231.

17. G. Hinton and R. Salakhutdinov, 2006, "Reducing the dimensionality of data with neural networks," Science Magazine, vol 313, pp. 504-507.

18. A. OrtizGarcia, J. Munilla, J. M. Gorriz and J. Ramirez, 2016, "Ensembles of Deep Learning Architectures for the early diagnosis of Alzheimers Disease", International Journal of Neural Systems, 26:7, 1650025.

19. F. C. Morabito, M. Campolo, N. Mammone, M. Versaci, S. Franceschetti, F. Tagliavini, V. Sofia, D. Fatuzzo, A. Gambardella, A. Labate, L. Mumoli, G.G. Tripodi, S. Gasparini, V. Cianci, C. Sueri, E. Ferlazzo and U. Aguglia, 2017, "Deep Learning Representation from Electroencephalography of Early-stage Creutzfeldt -Jakob Disease and Features for Differentiation from Rapidly Progressive Dementia", International Journal of Neural Systems, vol. 27, no.2, 1650039 .

20. R. T. Schirrmeister, J. T. Springenberg and L. D. 
Fiederer, 2017, "Deep learning with convolutional neural networks for brain mapping and decoding of movement-related information from the human EEG", arxiv.org/abs/1703.05051.

21. H. Cecotti and A. Graser, 2011, "Convolutional Neural Networks for P300 detection with application to brain-computer interfaces", IEEE Trans. Pattern Analysis and Machine Intelligence, vol. 33, no. 3, pp. 433-445.

22. R. U. Acharya, S. L. Oh, Y. Hagiwara, J. H. Tan and H. Adeli, 2018, "Deep convolutional neural network for the automated detection of seizure using EEG signals", Computers in Biology and Medicine, vol. 92.

23. A. Antoniades, L. Spyrou, C. C. Took and S. Sanei, 2016, "Deep learning for epileptic intracranial EEG data", 2016 IEEE 26th International Workshop on Machine Learning for Signal Processing (MLSP), pp. $1-6$.

24. M. Koziarski and B. Cyganek, 2017, "Image Recognition with Deep Neural Networks in Presence of Noise Dealing with and Taking Advantage of Distortions", Integrated Computer-Aided Engineering, 24:4, pp. 337-350.

25. F. Agostinelli, M. R. Anderson and H. Lee, 2013, "Adaptive multi-column deep neural networks with application to robust image denoising", Proc. Advances Neural Information Processing Systems, pp. 1493-1501.

26. D. Ciresan, U. Meier and J. Schmidhuber, 2012, "Multi-column deep neural networks for image classification", Proc. IEEE Conf. CVPR, pp. 3642-3649.

27. P. L. Nunez, R. Srinivasan, A. F. Westdorp, R. S. Wijesinghe, D. M. Tucker, R. B. Silberstein and P. J. Cadusch, 1997, "EEG coherency I: Statistics reference electrode volume conduction Laplacians cortical imaging and interpretation at multiple scales", Electroencephalogr. Clin. Neurophysiol., vol. 103, no. 5, pp. 499-515.

28. P. Vincent, H. Larochelle, I. Lajoie, Y. Bengio and P. A. Manzagol, 2010, "Stacked denoising autoencoders: learning useful representations in a deep network with a local denoising criterion", Journal of Machine Learning Research, vol. 11, pp. 3371-3408.

29. L. Panait and S. Luke, 2005, "Cooperative multiagent learning: the state of the art", Autonomous Agents and Multi-Agent Systems, vol. 11, no. 3, pp. $387-434$.

30. J. Bergstra, O. Breuleux, F. Bastien, P. Lamblin, R. Pascanu, G. Desjardins, J. Turian, D. Warde-Farley and Y. Bengio., 2010, Theano: A CPU and GPU Math Expression Compiler, Proceedings of the Python for Scientific Computing Conference (SciPy).

31. U. R. Acharya, R. Yanti, J. W. Zheng, M. R. Krishnan, J. H. Tan, R. J. Martis and C. M. Lim, 2013, "Automated diagnosis of epilepsy using CWT, HOS and texture parameters", International Journal of Neural Systems, vol. 23, no 3.

32. A. Bultan, 1999, "A four-parameter atomic decomposition of chirplets", IEEE Trans. Signal Process., vol. 47, no. 3, pp. 731-745.

33. Y. LeCun, L. Bottou, G. B. Orr, and K. R. Mller, Springer, 1998, "Efficient backprop", Neural Networks: Tricks of the Trade, pp. 9-50.

34. A. Antoniades, L. Spyrou, D. M. Lopez, A. Valentin, G. Alarcon, S. Sanei and C. C. Took, 2017 "Detection of Interictal Discharges with Convolutional Neural Networks Using Discrete Ordered Multichannel Intracranial EEG", Trans. on Neural Systems and Rehabilitation Engineering, vol. 25, no. 12, pp. 2285-2294. 